\title{
The Research of an Attribute Mathematical Model on Aquifer Water-abundance Evaluation
}

\author{
Dekang Zhao, Yang Wang, Jianjun Shen, Wenping Mu, Honglei Liu \\ (College of Geosciences and Surveying Engineering, China University of Mining and \\ Technology(Beijing),Beijing,100083,China) \\ zhaodekay@163.com
}

\begin{abstract}
Keywords: Water-abundance; Evaluation; Attribute Mathematical Theory; Similar Weight Abstract. The aquifer water-abundance evaluation and classification play an important role in the prevention and control of coal mine water disasters. Based on attribute mathematical theory and similar coefficient determining method, this paper propose an attribute mathematical systematically model for the aquifer water-abundance evaluation . This methodology consists of three parts: single index attribute measure analysis, multiple indices attribute measure analysis and attribute recognition analysis. By analyzing the attribute of every single factor index and multiple factor indices measurement, coal seam floor aquifer water-abundance in the study area has been divided into four grades: weak, medium, strong and extremely strong, which has great guiding significance for practical production.
\end{abstract}

\section{Introduction}

Mine water disaster is a common issue that constrained the development of the coal mine. Evaluating the Coal seam roof and floor water disasters risk by predicting and evaluating the aquifer water-abundance make great sense to the liberation of coal reserves threatened by mine water.

To partition the aquifer water-abundance, many traditional methods are proposed, such as single index method, comprehensive indices method, geological statistics method and so on. However, as varies complicated geological conditions in different areas, these methods have a lot of restrictions in the practical application. Even worse , the indices evaluation based on these traditional methods may result in contradictory conclusion problems. To overcome this problem, many scholars introduce some mathematical methods ,such as artificial neural network, fuzzy mathematic theory, grey system theory . However, each mathematical method has its own restriction. For instance, fuzzy mathematic theory adopts subjective weight method which make the weight great subjectivity.

The aquifer water-abundance partition is a qualitative measurement problem and is the recognition of orderly divided classes. Attribute mathematical theory just provides a better way on this problem. Attribute mathematical theory is a new method of comprehensive evaluation proposed by professor Qiansheng cheng in the 1990s, which is based on the conception of attribute set, attribute measurement space and ordered partition classification and so on. Confidence criterion is put forward according to the characteristics of the evaluation set-ordering.

In this paper, an aquifer water-abundance evaluation model is established based on attribute mathematical theory, which provide an effective approach for the evaluation and prediction of Coal seam roof and floor water disasters risk.

\section{Attribute mathematical model}

Attribute mathematical model is mainly composed of three parts: single index attribute measure analysis, multiple index attribute measure analysis, attribute recognition analysis.

Assume one of the evaluation factor $x_{i}$ need to measure $m$ indices $I=\left(I_{1}, I_{2}, \cdots, I_{m}\right)$. For every measurement $t_{i j}$ of index $I_{j}$ in $x_{i}$, there are $p$ evaluation grades $C_{k}(k=1,2, \cdots, p)$. Thus the grade of $t_{i j}$ can be determined. 
Single Index Attribute Measure Analysis. The measured value $t_{i j}$ of the single index $I_{j}$ belongs to the attribute grade $C_{k}$. The determination method of attribute measure value $\mu_{x j k}$ is establishing the attribute measure function, which usually expressed in the form of Table 1.

Table 1 Grade standards of variable indices

\begin{tabular}{ccccc}
\hline Evaluation & \multicolumn{4}{c}{ Evaluation grades } \\
\cline { 2 - 5 } indices & $C_{1}$ & $C_{2}$ & $\ldots$ & $C_{k}$ \\
\hline$I_{1}$ & $a_{10} \sim a_{11}$ & $a_{11} \sim a_{12}$ & $\ldots$ & $a_{1 k-1} \sim a_{1 k}$ \\
$I_{2}$ & $a_{20} \sim a_{21}$ & $a_{21} \sim a_{22}$ & $\ldots$ & $a_{2 k-1} \sim a_{2 k}$ \\
$\vdots$ & $\vdots$ & $\vdots$ & $\vdots$ & $\vdots$ \\
$I_{m}$ & $a_{m 0} \sim a_{m 1}$ & $a_{m 1} \sim a_{m 2}$ & $\ldots$ & $a_{m k-1} \sim a_{m k}$ \\
\hline
\end{tabular}

In Table 1, $a_{j k}$ satisfy $a_{j 0<} a_{j 1<\cdots<} a_{j \mathrm{~K}}$ or $a_{j 0>} a_{j 1>\cdots>} a_{j \mathrm{~K}}$. Based on the assumption of $a_{j 0<} a_{j 1<\cdots<} a_{j \mathrm{~K}}$, parameters and are defined as:

$$
b_{j k}=\frac{a_{j k-1}+a_{j k}}{2} ; \quad d_{j k}=\min \left(\left|b_{j k}-a_{j k}\right|,\left|b_{j k+1}-a_{j k}\right|\right) .
$$

The index of the evaluation object $X$ ranked $j$ is defined as $t$, then the single index attribute measure function $\mu_{x j k}(t)$ can be calculated by the functions in Table 2 .

Table 2 Single Index Attribute Measure Functions

$$
\mu_{j k}(t)=\left\{\begin{array}{cc}
0 & t<a_{j k-1}-d_{j k-1} \\
\frac{\left|t-a_{j k-1}+d_{j k-1}\right|}{2 d_{j k-1}} & a_{j k-1}-d_{j k-1} \leqslant t \leqslant a_{j k-1}+d_{j k-1} \\
1 & a_{j k-1}+d_{j k-1}<t<a_{j k}-d_{j k} \\
\frac{\left|t-a_{j k}-d_{j k}\right|}{2 d_{j k}} & a_{j k}-d_{j k} \leqslant t \leqslant a_{j k}+d_{j k} \\
0 & t>a_{j k}+d_{j k}
\end{array} \quad \mu_{j k}(t)=\left\{\begin{array}{cc}
1 & t<a_{j 1}-d_{j 1} \\
\frac{\left|t-a_{j 1}-d_{j 1}\right|}{2 d_{j 1}} & a_{j 1}-d_{j 1} \leqslant t \leqslant a_{j 1}+d_{j 1} \\
o & t>a_{j 1}+d_{j 1}
\end{array} \quad \mu_{j k k}(t)=\left\{\begin{array}{cc}
1 & t<a_{j 1}-d_{j 1} \\
\frac{\left|t-a_{j 1}-d_{j 1}\right|}{2 d_{j 1}} & a_{j 1}-d_{j 1} \leqslant t \leqslant a_{j 1}+d_{j 1} \\
0 & t>a_{j 1}+d_{j 1}
\end{array}\right.\right.\right.
$$

Multiple Indices Attribute Measure Analysis. The multiple indices attribute measure value can be expressed as:

$$
\mu_{x k}=\sum_{j=1}^{m} \omega_{j} \mu_{x j k} \cdot
$$

where $\omega_{j}$ is the weight of evaluation index $I_{j}$. The weight $\omega_{j}$ can be determined by defining the similar weight, which was calculated through the similar coefficient. Firstly, assume the weight of every index is $\omega_{j}=1 / m$, then the similar coefficient $r_{j}$ and similar weight $\omega_{j}$ of attribute measurement evaluation matrix can be expressed in the following forms:

$$
\begin{aligned}
& r_{j}=\frac{1}{n} \sum_{i=1}^{n}\left(\mu_{i j 1}, \mu_{i j 2}, \cdots, \mu_{i j k}\right) \cdot\left(\mu_{i 1}, \mu_{i 2}, \cdots, \mu_{i k}\right)^{\mathrm{T}}=\frac{1}{n} \sum_{i=1}^{n} \sum_{k=1}^{K} \mu_{i j k} \cdot \mu_{i k} \cdot \\
& \omega_{j}=r_{j} / \sum_{j=1}^{m} r_{j} .
\end{aligned}
$$


The aim of attribute recognition analysis is that according to the attribute measure value $\mu_{x k}$ determining which evaluation grade the evaluation object $x$ belongs to. The main gist of this determination is the confidence criterion:

The evaluation set $\left(C_{1}, C_{2}, \cdots, C_{k}\right)$ is an ordered set and it is assumed that $C_{1}<C_{2}<\cdots<C_{k}$, if

$$
k_{0}=\max \left\{k: \sum_{l=k}^{p} \mu_{x l} \geqslant \lambda, 1 \leqslant k \leqslant p\right\} .
$$

the evaluation object $x$ is determined as grade $C_{k 0}$. In the Eq. (5), $\lambda$ is the confidence coefficient, and generally ranges from 0.6 to 0.7 .

\section{Application}

The factors influencing the water-abundance of aquifer are manifold.In this paper, four indices are selected to evaluate the water-abundance: aquifer thickness, flush fluid loss, core recovery of drilling hole and brittle-plastic rock ratio. Applying the attribute measure theory, the aquifer water-abundance is divided into four grades: weak, medium, strong and extremely strong(Table 3).

Table 3 Water-abundance Evaluation Index System and Hierarchy

\begin{tabular}{lcccc}
\hline \multirow{2}{*}{ Evaluation indices } & \multicolumn{4}{c}{ Water-abundance grades } \\
\cline { 2 - 5 } & $C_{1}$ (weak) & $C_{2}$ (medium) & $C_{3}$ (strong) & $C_{4}$ (extremely strong) \\
\hline $\mathrm{U}_{1}$ : Aquifer thickness $(\mathrm{m})$ & $<10$ & $10 \sim 15$ & $15 \sim 20$ & $>20$ \\
$\mathrm{U}_{2}$ : Flush fluid loss $\left(\mathrm{m}^{3} / \mathrm{h}\right)$ & $<0.15$ & $0.15 \sim 0.25$ & $0.25 \sim 0.35$ & $>0.35$ \\
$\mathrm{U}_{3}$ : Core recovery & $>0.8$ & $0.8 \sim 0.65$ & $0.65 \sim 0.5$ & $<0.5$ \\
$\mathrm{U}_{4}$ : Brittle-plastic rock ratio & $>20$ & $20 \sim 15$ & $15 \sim 10$ & $<10$ \\
\hline
\end{tabular}

According to the single index attribute measure function $\mu_{x j k}(t)$ and the Table 3 , take the $U_{1}$ ( aquifer thickness) for example ,the single index attribute measure functions are built as Table 4 .

Table 4 Single Index Attribute Measure Functions For $\mathrm{U}_{1}$

\begin{tabular}{|c|c|c|c|c|c|c|c|c|c|c|c|}
\hline \multicolumn{3}{|c|}{$C_{1}$} & \multicolumn{3}{|c|}{$C_{2}$} & \multicolumn{3}{|r|}{$C_{3}$} & \multicolumn{3}{|c|}{$C_{4}$} \\
\hline$\mu_{x 11}=$ & $\left\{\begin{array}{c}1 \\
\frac{12.5-t}{5} \\
0\end{array}\right.$ & $\begin{array}{c}t<7.5 \\
7.5 \leqslant t \leqslant 12.5 \\
t>12.5\end{array}$ & $\mu_{x 12}=$ & $\left\{\begin{array}{c}0 \\
\frac{t-7.5}{5} \\
\frac{17.5-t}{5} \\
0\end{array}\right.$ & $\begin{array}{c}t<7.5 \\
7.5 \leqslant t \leqslant 12.5 \\
12.5<t<17.5 \\
t \geqslant 17.5\end{array}$ & $\mu_{x 13}=$ & $\left\{\begin{array}{c}0 \\
\frac{t-12.5}{5} \\
\frac{22.5-t}{5} \\
0\end{array}\right.$ & $\begin{array}{c}t<12.5 \\
12.5 \leqslant t \leqslant 17.5 \\
17.5<t<22.5 \\
t \geqslant 22.5\end{array}$ & $\mu_{x 14}=$ & $\left\{\begin{array}{c}0 \\
t-17.5 \\
5 \\
1\end{array}\right.$ & $\begin{array}{c}t<17.5 \\
17.5 \leqslant t \leqslant 22.5 \\
t>22.5\end{array}$ \\
\hline
\end{tabular}

The measured data of Permian sandstone aquifer of 2 \# coal roof of 9103 working face in a coal mine in Shanxi Province are showed in the Table 5.

Table 5 Measured Data

\begin{tabular}{lllll}
\hline Study area & $\begin{array}{l}\text { Aquifer } \\
\text { thickness }(\mathrm{m})\end{array}$ & $\begin{array}{l}\text { Flush fluid } \\
\operatorname{loss}\left(\mathrm{m}^{3} / \mathrm{h}\right)\end{array}$ & $\begin{array}{l}\text { Core } \\
\text { recovery }\end{array}$ & $\begin{array}{l}\text { Brittle-plastic } \\
\text { rock ratio }\end{array}$ \\
\hline 9102 working face & 18.6 & 0.26 & 0.85 & 16.5 \\
\hline
\end{tabular}

Then the Single index attribute measure evaluation matrix can be calculated as: 
$\eta=\left[\begin{array}{cccc}0 & 0 & 0.78 & 0.22 \\ 0 & 0.4 & 0.6 & 0 \\ 0.83 & 0.17 & 0 & 0 \\ 0 & 0.8 & 0.2 & 0\end{array}\right]$

The similar coefficient, similar weight and Multiple index attribute measure matrix of the evaluation can be obtained by using the Eqs.(3) (5):

$r_{j}=(0.5655,0.4833,0.3288,0.51) ; \omega_{j}=(0.2996,0.256,0.1742,0.2702) ; \mu_{x k}=[0.145,0.348,0.441,0.066]$.

When $\lambda=0.65, k_{0}=\max \left\{k: \sum_{l=k}^{4} \mu_{x l} \geqslant 0.65,1 \leqslant k \leqslant 4\right\}$.

Obviously, when $\mathrm{k}=3$ or 4 , the inequality is satisfied, so $k_{0}=3$, namely the water-abundance grade is $C_{3}$ and the study area has strong water abundance.

\section{Conclusion}

(1) According to the hydrogeological conditions in the study area, four indices are selected to evaluate the water-abundance: aquifer thickness, flush fluid loss, core recovery of drilling holes and brittle-plastic rock ratio. And then aquifer water-abundance evaluation model is established based on attribute mathematical theory.

(2)The model is applied in an sandstone aquifer of coal roof in a coal mine in Shanxi Province, which shows that the method provides an effective approach for the water-abundance evaluation.

\section{References}

[1] Qiansheng Cheng: Acta Scientiarum Naturalism University pekinensis , 1997, 33(1): 12-20.(in chinese)

[2] Shu-cai Li, Zong-qing: Tunneling and Underground Space Technology, 2013, 38(1): 50-58.

[3] Yan-kun Ma, En-yuan Wang, Zhen-tang Liu: Journal of Mining \&Safety Engineering, 2012, 29(3): (416-420). (in chinese)

[4] L.P.Li, Z.Q.Zhou, S.C.Li: Mine Water and the Environment, 2015, 34(3): 288-294.

[5] Zong-qing Zhou, Shu-cai Li: Rock and Soil Mechanics, 2013, 34(3):819-826.( in chinese) 